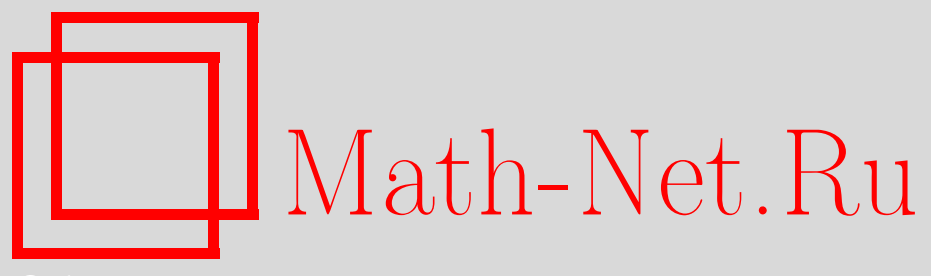

О. Н. Карпенков, О критерии существования и единственности целочисленного треугольника с заданными углами, УМH, 2006, том 61, выпуск 6, 185-186

DOI: https://doi.org/10.4213/rm5395

Использование Общероссийского математического портала Math-Net.Ru подразумевает, что вы прочитали и согласны с пользовательским соглашением http://www . mathnet.ru/rus/agreement

Параметры загрузки:

IP : 18.209 .158 .208

26 апреля 2023 г., 18:32:29

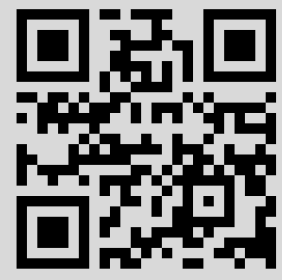




\section{О критерии существования и единственности целочисленного треугольника с заданными углами}

\section{О. Н. Карпенков}

Проблема описания выпуклых целочисленных многоугольников в терминах целочисленных инвариантов является нерешенной. На настоящий момент известно только, что число выпуклых целочисленных многоугольников с ограниченной площадью $n$ растет экспоненциально по $n^{1 / 3}$ (см. работы [1] и [2]). В этой заметке полностью исследован случай треугольников. Автор благодарен В. И. Арнольду, И. Барани и А. Г. Хованскому за внимание к этой работе и полезные замечания.

Общие определения. Рассмотрим двумерную ориентированную вещественную аффинную плоскость. Зафиксируем в этой плоскости некоторую систему координат $O X Y$. Точка плоскости называется целой, если все ее координаты целые. Выпуклая оболочка конечного числа целых точек, не содержащихся в одной прямой, называется иелочисленным выпуклым многоугольником. Рассмотрим минимальный набор точек, определяющий заданный многоугольник. Точки этого набора называются вериинами многоугольника. Поскольку все вершины лежат на границе выпуклой оболочки, их можно циклически упорядочить против или по ходу часовой стрелки: $A_{1}, \ldots, A_{n}$. Назовем такие многоугольники положительно-ориентированными и отрицательно-ориентированными соответственно и обозначим $A_{1} \ldots A_{n}$.

Назовем углом упорядоченный набор двух замкнутых лучей с общей вершиной, не содержащихся в одной прямой. Лучи называются сторонами угла, а их общая вершина - вершиной угла. Угол называется целочисленным, если его вершина является целой, а обе стороны содержат целые точки, отличные от вершины. Углом $\angle A B C$ ориентированного целочисленного многоугольника с последовательными вершинами $A, B$ и $C$ называется целочисленный угол с вершиной $B$ и сторонами $B A$ и $B C$.

Аффинное преобразование плоскости называется челочисленно-аффинным, если оно сохраняет множество целых точек. Многоугольники $A_{1} \ldots A_{n}$ и $B_{1} \ldots B_{n}$ (углы $\angle A_{1} A_{2} A_{3}$ и $\left.\angle B_{1} B_{2} B_{3}\right)$ называются целочисленно-эквивалентными, если существует целочисленно-аффинное преобразование плоскости, переводящее точки $A_{i}$ в $B_{i}$, где $i=1, \ldots, n$ (соответственно лучи $A_{2} A_{1}$ и $A_{2} A_{3}$ в лучи $B_{2} B_{1}$ и $B_{2} B_{3}$ ).

Для положительного целого $n$ и точки $A(x, y)$ обозначим через $n A$ точку с координатами $(n x, n y)$. Многоугольник $n A_{0} \ldots n A_{k}$ называется $n$-гомотетичным многоугольнику $P=A_{0} \ldots A_{k}$ и обозначается через $n P$. Многоугольники $P_{1}$ и $P_{2}$ называются целочисленно-подобными, если существуют целые положительные числа $m_{1}$ и $m_{2}$ такие, что $m_{1} P_{1}$ целочисленно-эквивалентно $m_{2} P_{2}$.

Конечные цепные дроби. Дополним множество рациональных чисел с операциями + и $1 / *$ элементом $\infty$ и обозначим: $\overline{\mathbb{Q}}$. Положим $q \pm \infty=\infty, 1 / 0=\infty, 1 / \infty=0$ (выражения $\infty \pm \infty$ не определены).

Любой конечной последовательности целых чисел $\left(a_{0}, a_{1}, \ldots, a_{n}\right)$ поставим в соответствие элемент $a_{0}+1 /\left(a_{1}+1 /\left(a_{2}+\cdots+1 /\left(a_{n-1}+1 / a_{n}\right) \cdots\right)\right)$ множества $\overline{\mathbb{Q}}$ и обозначим его $] a_{0}, a_{1}, \ldots, a_{n}\left[\right.$. Если числа последовательности $a_{1}, \ldots, a_{n}$ - положительные, то запись для $q$ называется обыкновенной цепной дробъю.

ПреДЛОЖЕНИЕ. Для любого рационального числа существует и единственна обыкновенная чепная дробъ с нечетным числом элементов.

Работа выполнена при поддержке грантов НШ-1972.2003.1, NWO-RFFI 047.011.2004.026 (РФФИ 05-02-89000-NWO_а), РФФИ 05-01-02805-CNRSL_а и РФФИ-05-01-01012а. 
Рассмотрим для $q_{i} \in \mathbb{Q}, i=1, \ldots, k$, обыкновенные цепные дроби с нечетным числом элементов: $\left.q_{i}=\right] a_{i, 0}, a_{i, 1}, \ldots, a_{i, 2 n_{i}}[$. Обозначим через $] q_{1}, q_{2}, \ldots, q_{k}[$ элемент

$$
] a_{1,0}, a_{1,1}, \ldots, a_{1,2 n_{1}}, a_{2,0}, a_{2,1}, \ldots, a_{2,2 n_{2}}, \ldots, a_{k, 0}, a_{k, 1}, \ldots, a_{k, 2 n_{k}}[\in \overline{\mathbb{Q}} .
$$

Целочисленные тангенсы. Целочисленной длиной отрезка $A B$ (обозначение $1 \ell(A B))$ называется отношение его евклидовой длины к минимальной евклидовой длине целочисленного вектора с концами в отрезке АВ. Целочисленной (неориентированной) площадъю многоугольника $P$ (обозначение $\mathrm{IS}(P))$ называется удвоенная евклидова площадь многоугольника.

Рассмотрим произвольный целочисленный угол $\angle A B C$. Граница выпуклой оболочки множества всех целых точек, за исключением $B$, входящих в выпуклую оболочку угла $\angle A B C$, называется парусом ортанта. Парус угла представляет собой конечную ломаную линию, с началом и концом на разных сторонах угла. Ориентируем ломаную от луча $B A$ к лучу $B C$ и обозначим ее вершины: $A_{0}, \ldots, A_{m+1}$. Обозначим $a_{i}=1 \ell\left(A_{i} A_{i+1}\right)$ при $i=0, \ldots, m$, а также $b_{i}=\operatorname{lS}\left(A_{i-1} A_{i} A_{i+1}\right)$ при $i=1, \ldots, m$. Целочисленным тангенсом угла $\angle A B C$ называется следующее рациональное число:

$$
] a_{0}, b_{1}, a_{1}, b_{2}, a_{2}, \ldots, b_{m}, a_{m}[\quad \text { (обозначение: } \operatorname{ltg} \angle A B C) .
$$

Формулировка теоремы. В евклидовой геометрии на плоскости условие на существование треугольника с заданными углами переписывается через тангенсы углов следующим образом. Треугольник с углами $\alpha, \beta$ и $\gamma$ существует тогда и только тогда, когда $\operatorname{tg}(\alpha+\beta+\gamma)=0$ и $\operatorname{tg}(\alpha+\beta) \notin[0 ; \operatorname{tg} \alpha]$ (здесь, не нарушая общности, мы предполагаем, что $\alpha$ - острый). Приведем целочисленный аналог последнего утверждения.

Теорема. а) Пусть $\alpha_{0}, \alpha_{1} u \alpha_{2}$ - упорядоченная тройка целочисленных уалов. Ориентированный целочисленный треугольник с последовательными углами, целочисленно-эквивалентными углам $\alpha_{0}, \alpha_{1}$ и $\alpha_{2}$, существует тогда и только тогда, когда существует $j \in\{1,2,3\}$ такое, что углы $\alpha=\alpha_{j}, \beta=\alpha_{j+1(\bmod 3)} u \gamma=$ $\alpha_{j+2(\bmod 3)}$ удовлетворяют следующим условиям:

i) $] \operatorname{ltg} \alpha,-1, \operatorname{ltg} \beta,-1, \operatorname{ltg} \gamma[=0 ; \quad$ ii) $] \operatorname{ltg} \alpha,-1, \operatorname{ltg} \beta[\notin[0 ; \operatorname{ltg} \alpha]$;

б) два челочисленных треугольника, последовательности целочисленных тангенсов которых совпадают, являются целочисленно-подобными.

Отметим, что в условии теоремы для тангенсов углов всегда берутся обыкновенные цепные дроби с нечетным числом элементов. Проиллюстрируем теорему на следующем частном примере:

$$
\begin{aligned}
& \operatorname{ltg} \alpha=3=] 3[; \\
& \operatorname{ltg} \beta=9 / 7=] 1,3,2[; \\
& \operatorname{ltg} \gamma=3 / 2=] 1,1,1[;
\end{aligned}
$$

i) $] 3,-1,1,3,2,-1,1,1,1[=0$;

ii) $] 3,-1,1,3,2[=-3 / 2 \notin[0 ; 3]$.

\section{Список литературы}

[1] В. И. Арнольд, Функи. анализ и его прил., 14:2 (1980), 1-3. [2] I. Bárány, A. M. Vershik, Geom. Funct. Anal., 2:4 (1992), 381-393.

O. H. Карпенков (O. N. Karpenkov)

Независимый московский университет

E-mail: karpenk@mccme.ru
Представлено А. Б. Сосинским Принято редколлегией 14.08.2006 